\section{Arthritis and HLA-B27 in Papua New Guinea}

Visitors to the hospitals of Papua New Guinea are often surprised by the many beds occupied by young men with an asymmetrical arthritis, predominantly affecting the legs. Scrutiny of their notes often elicits the enigmatic term "tropical arthritis." Such patients have been the subject of several studies. Maddocks came down firmly in favour of Reiter's disease as the diagnosis in most of these cases. ${ }^{1} \mathrm{~A}$ later study from Goroka Base Hospital found no extra-articular manifestations of Reiter's disease and concluded that the disease was a distinctive type of arthritis of unknown aetiology. ${ }^{2}$ Subsequently Brewerton et al described a close link between HLA-B27 and Reiter's disease. ${ }^{3}$ HLA studies in patients with arthritis in Papua New Guinea have not previously been published.

\section{Patients, methods, and results}

We studied 64 patients with arthritis, who presented to the physician (JER) at Goroka Base Hospital between October 1984 and March 1985. During this period only four other patients with arthritis were seen (two with gout, one with osteoarthritis, and one with rheumatic fever). All patients were examined by JER. Sixty two were of highland origin and two were from the coast. Histocompatibility testing was done by the procedure outlined by Bhatia $e t$ al. The frequency of HLA antigens in the patients was compared with that in 128 Papua New Guinean blood donors matched for age, sex, and province of origin (two controls per patient).

HLA-B27 was found in $45(70 \%)$ of the 64 patients and in $16(13 \%)$ of the 128 controls. It was present in all 10 patients with the classic triad of symptoms of Reiter's disease and in $13(87 \%)$ of the 15 patients with less complete forms of Reiter's disease (table). Information about sexual exposure before arthritis was not readily obtained. Shigella flexneri serotype I had been isolated from one patient during an earlier admission for dysentery. Keratoderma blennorrhagicum and other mucocutaneous lesions (except balanitis) were not seen. HLA-B27 was noted in $22(56 \%)$ of the 39 patients with symptoms confined to joints.

\section{Comment}

HLA-B27 has been reported as rare or absent among several peoples in the south west Pacific. The incidence of HLA-B27 (13\%) in our controls was close to that in Europeans. The strong association of HLA-B27 with arthritis in our patients vindicates Maddocks's view that Reiter's disease or something closely akin to it is the predominant form of arthritis in Papua New Guinea. ${ }^{1}$ The failure of Jeremy et al to recognise Reiter's disease in their study $^{2}$ might indicate a recent spread of Reiter's disease into the highlands or might reflect difficulties in obtaining accurate clinical details.

Our series lends further support to the hypothesis that HLA-B27 is linked to a range of rheumatic disorders that includes Reiter's disease, reactive arthritis, and other forms of arthritis. ${ }^{5}$ Arthritis associated with HLA-B27 is unusually common in Papua New Guinea, but, interestingly, ankylosing spondylitis is remarkably rare. We have little idea which of the infections linked to Reiter's disease and reactive arthritis will prove to be important locally. Sexually transmitted disease and shigellosis are both widespread; no data are available on chlamydial infections.

This study was partly financed by the Papua New Guinea Biological Foundation, who gave a grant to KB.

Maddocks I. Reiter s disease in Port Moresby, Papua. Br f V'ener Dis 1967;43:280-3.

2 Jeremy R. Rhodes FA, Sharp JT, Rawls WE. Clinical and laboratory studies of a distinctive type of arthritis observed in New Guinea. Med $\mathcal{F}$ Aust 1969:j:1273-9.

Brewerton DA, Caffrey M, Nicholls A, Walters D, Oates JK, James DCO. Reiter's disease and HL-A 27. Lancet 1973;ii:996-8.

Bhatia K, Gorogo M, Koki G. HLA-A, B, C and DR antigens in Asaro speakers of Papua New Guinea. Hum Immunol 1984;9:189-200.

5 Arnett FC, McCluskey OE, Schacter BZ, Lordon RE. Incomplete Reiter's syndrome: discriminating features and HL-A W27 in diagnosis. Ann Intern Med 1976;84:8-12.

Accepted 4 August 1986

Goroka Base Hospital, PO Box 392, Goroka, Eastern Highlands Province, Papua New Guinea

J E RICHENS, MSC, MRCP, specialist medical officer (physician)

Papua New Guinea Institute of Medical Research, PO Box 60, Goroka M L PRASAD, MSC, MPHIL, research officer K BHATIA, PHD, senior research fellow

Faculty of Medicine, Hong Kong University

M TUNG, medical student

Correspondence and requests for reprints to: Dr Richens.

\section{Acquired cystic disease of the kidney: an indication for renal transplantation?}

Acquired cystic disease of the kidney is common in patients receiving dialysis and may be associated with important clinical complications. ${ }^{12}$ There is little information on the disease in recipients of renal transplants. We compared the prevalence of cystic disease in patients with a functioning renal transplant and in patients with end stage renal disease treated by haemodialysis and chronic ambulatory peritoneal dialysis.

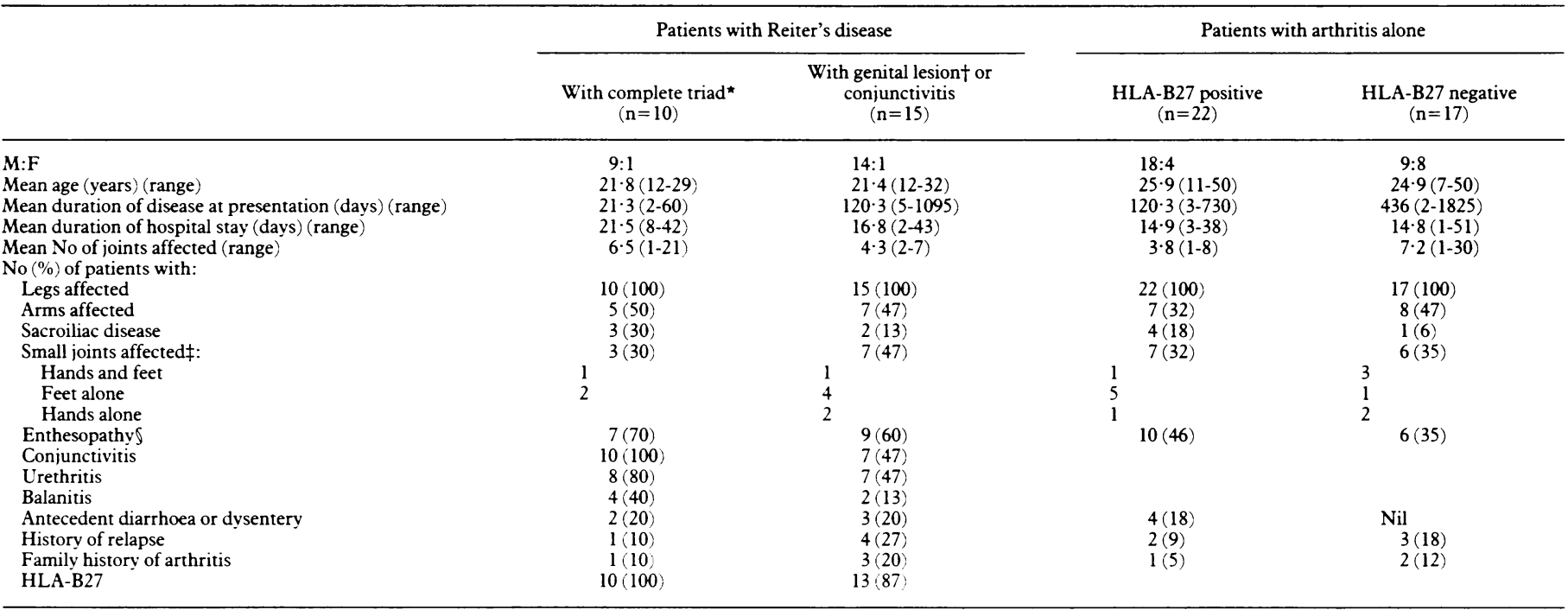

ॠComplete triad $=$ arthritis, conjunctivitis, and genital lesion. $\dagger$

tGenital lesion = urethritis or balanitis, or both.

†Tender metacarpophalangeal, metatarsophalangeal, and interphalangeal joints, or "sausage digits."

$§$ Tenderness over tibial tuberosity, tendo Achillis, os calcis, or deltoid ligament. 


\section{Patients, methods, and results}

We studied 45 patients aged 18-72 (mean 47) who had had a functioning transplant for one to 14 (mean $4 \cdot 2$ ) years and 100 patients aged $18-78$ (mean 51 ) who had been receiving dialysis for one to 21 (mean 4.8 ) years. There were no significant differences between the two groups in age, sex, or nature of underlying disease. No patient had polycystic kidney disease of adult onset. Thirty two of the patients receiving dialysis were receiving chronic ambulatory peritoneal dialysis. In the transplant group the mean total duration of renal replacement treatmentthat is, the duration of dialysis plus the duration of the transplant - was 6.7 years and the mean duration of dialysis before transplantation 2.5 years.

Each patient underwent real time ultrasonography performed by one of two experienced radiologists, who used ultrasound equipment of identical resolution. Each scan was assessed by one radiologist without reference to details of the patient and graded $0-4$ according to the number of cysts per kidney $(0=$ no cysts, $1=<5,2=5-9,3=10-14$, and $4=\geqslant 15$ )

Eighteen patients $(40 \%)$ in the transplant group had acquired cystic disease of their native kidneys ( 14 grade 1 , two grade 2 , and two grade 3 ). No cysts were detected in any transplanted kidney. Sixty three patients $(63 \%)$ receiving dialysis had acquired cystic disease ( 24 grade 1,14 grade 2, 10 grade 3 , and 15 grade 4 ). One patient receiving dialysis had a solid lesion in one kidney that proved to be renal adenocarcinoma. There was no correlation in the transplant group between the number of cysts and the total duration of renal replacement treatment (Kendall rank correlation) (figure). In addition, there was no correlation with

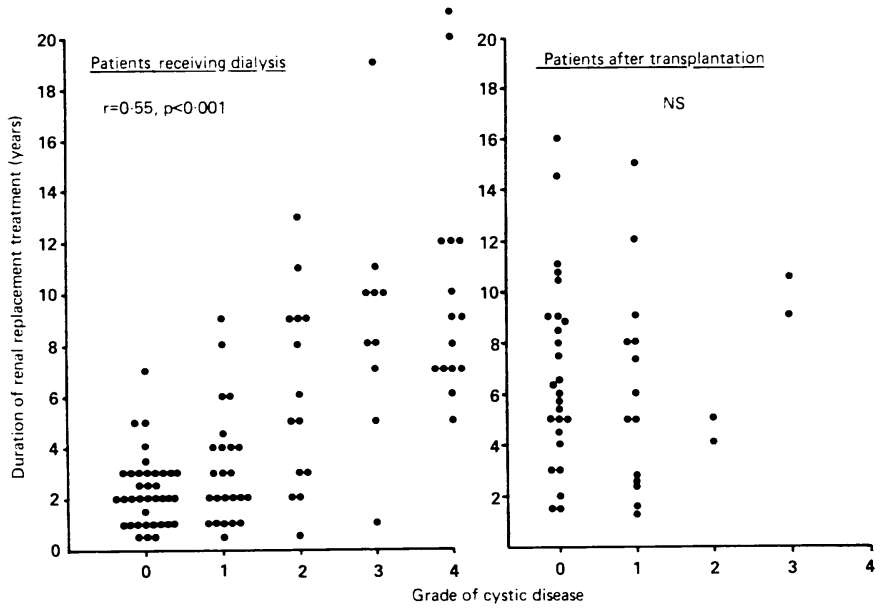

Relation between grade of cystic disease and duration of renal replacement treatment in group receiving dialysis and group after transplantation.

either the duration of the transplanted kidney or the duration of dialysis before transplantation. There was a highly significant correlation between the number of cysts and the duration of dialysis in the dialysis group $(\mathrm{p}<0 \cdot 001$, Kendall rank correlation) (figure). Within this group there were no significant differences between those treated by haemodialysis and those treated by chronic ambulatory peritoneal dialysis.

\section{Comment}

This study showed that acquired cystic disease of the kidneys progresses in patients treated by dialysis alone but not in patients with a functioning renal transplant. In addition, the lack of correlation between the number of cysts and the duration of dialysis before transplantation suggests that the disease may regress after successful transplantation. Cystic disease is an important complication of both haemodialysis and chronic ambulatory peritoneal dialysis and is likely to increase in prevalence as the number of patients receiving long term dialysis grows. It can cause intrarenal and extrarenal haemorrhage, which may be fatal. ${ }^{23}$ The most important complication, however, is an apparently greatly increased risk of neoplasm arising in kidneys that have undergone multicystic transformation. Gardner calculated that patients with cystic disease are 14 times more likely to develop renal adenocarcinoma than patients with chronic renal failure without cystic disease. ${ }^{+}$We detected one neoplasm in this study, and in addition we previously reported on a patient with acquired polycystic disease who died from metastatic renal adenocarcinoma. ${ }^{5}$ Our finding that acquired polycystic disease of the kidneys is much less severe after transplantation may therefore be an important consideration in the choice of long term renal replacement treatment.

1 Grantham JJ, Levine E. Acquired cystic disease: replacing one kidney disease with another. Kidney Int 1985;28:99-105.

2 Ratcliffe PJ, Dunnill MS, Oliver DO. Clinical importance of acquired cystic disease of the kidney in patients undergoing dialysis. Br.Med f 1983;287:1855-8.
3 Feiner HD, Katz LA, Gallo GR. Acquired cystic disease of the kidney in chronic dialysis patients. Urology 1981;18:260-4.

4 Gardner KD. Acquired renal cystic disease and renal adenocarcinoma in patients on long term heamodialysis. NEngl f Med 1984;310:390.

5 Thompson BJ, Allan PL, Winney RJ. Acquired cystic disease of the kidney: metastatic renal adenocarcinoma and hypercalcaemia. Lancet $1985 ;$;i: $502-3$.

Accepted 28 fuly 1986)

\section{Royal Infirmary, Edinburgh EH3 9YW}

B J THOMPSON, MRCP, registrar, medical renal unit

D A S JENKINS, MRCP, registrar, medical renal unit

P L ALLAN, FRCR, honorary consultant, department of radiology

R J WINNEY, FRCP, consultant renal physician, medical renal unit

\section{Western General Hospital, Edinburgh}

J C B DICK, MRCP, registrar, Nuffield transplantation surgical unit

S R WILD, FRCR, consultant radiologist

J L ANDERTON, FRCP, consultant nephrologist, Nuffield transplantation surgical unit

G D CHISHOLM, FRCS, professor of urology, Nuffield transplantation surgical unit

Correspondence to: Dr Thompson.

\section{Severe illness associated with appearance of antibody to human immunodeficiency virus in an African}

Encephalopathy and acute illnesses similar to mononucleosis associated with the appearance in serum of antibody to human immunodeficiency virus $(\mathrm{HIV})^{1-3}$ have not previously been reported in Africa.

\section{Case report}

A previously healthy 35 year old African man became ill in November 1984 (day 0 ) with vague malaise and fatigue. The next day he developed joint pains and fever $\left(39^{\circ} \mathrm{C}\right)$, which persisted over the next 13 days. Additional problems noted on day 2 included pharyngitis, diarrhoea, and vomiting and a pruritic maculopapular rash on the face. By day 3 the rash extended to the trunk and occipital ymphadenopathy was noted. On day 4 shallow ulcers were observed on the palatal and gingival mucosa and on the penis and scrotum. He was admitted and during the next five days became progressively obtunded to the extent that he was unable to speak or recognise people; this state persisted until the 15 th day, when all his symptoms dramatically improved. He was released from hospital on day 19. Antibiotics (penicillin analogues and tetracycline) were given from day 2 but ho apparent effect. During the three weeks after discharge his condition continued to improve but he developed generalised lymphadenopathy; biopsy showed only non-specific reactive hyperplasia. He resumed work at nine weeks At his last visit (14 months) he was working full time and had regained all lost weight. The lymphadenopathy had gradually lessened from the end of the fourth month but was still present.

The results of Western blot analyses (figure) and enzyme linked immunosorbent assay clearly showed that antibody to HIV was present in the serum by day 11 . The leucocyte count was initially $5.0 \times 10^{\circ} / 1$ (25\% lymphocytes) and gradually rose to $12.9 \times 10^{9} / 1$ (29\% lymphocytes) by the day of discharge. From day 11 onward, coincident with the development of antibodies, lymphocytes (up to $10 \%$ ) were consistently described as reactive. Results of liver function studies were abnormal during the second week of illness. Monospot tests and tests for febrile agglutinins yielded negative results, and the titres of antibodies against Epstein-Barr virus, cytomegalovirus, and toxoplasmosis in serum obtained before and after the illness were similar. Cerebrospinal fluid, examined twice during the second week, showed only slight lymphocytosis ( 10 cells and 22 cells) and no evidence of cryptococcus. Bacterial cultures and blood smears for malaria vielded negative results. There were no metabolic abnormalities. To detect viruses serum, cerebrospinal fluid, and scrapings from the oral ulcers collected during the acute illness were inoculated into suckling mice and on to Vero cell cultures, all with no effect. The ratio of T lymphocyte helper to suppressor cells at nine weeks was inverted $(0 \cdot 5)$, primarily because of a high proportion of suppressor cells ( $22 \%$ helpers, $42 \%$ suppressors; leucocyte count $6.9 \times 10^{9} / 1,68 \%$ lymphocytes). At seven months the ratio remained inverted $(0.85)$.

The patient did not know of any exposure to patients with the acquired immune deficiency syndrome or to needlesticks during the past year, denied homosexuality and drug abuse, and had never received a blood transfusion. $\mathrm{He}$ had not travelled outside Kenya since 1982. During the past year he had had many female sexual partners but no contact with prostitutes or venereal disease.

\section{Comment}

No other report has documented the acquisition of antibodies in relation to clinical symptoms in similar detail. Antibody against p24 and p55 